\title{
VI. LEG 42A PHYSICAL PROPERTIES DATA
}

\author{
Albert J. Erickson, Geology Department, University of Georgia, Athens, Georgia
}

TABLE 1

\begin{tabular}{ccc}
\hline $\begin{array}{c}\text { Sample (Interval } \\
\text { in cm) }\end{array}$ & $\begin{array}{c}\text { Subbottom } \\
\text { Depth (m) }\end{array}$ & $\begin{array}{r}\text { Sonic Velocity } \\
(\mathrm{km} / \mathrm{sec})\end{array}$ \\
\hline & & \\
Site 371 & & \\
& & \\
$1-4,10$ & 4.60 & 1.532 \\
$1-4,70$ & 5.20 & 1.546 \\
$1-4,100$ & 5.50 & 1.525 \\
$1-4,135$ & 5.85 & 1.544 \\
$2-3,30$ & 202.80 & 1.740 \\
$2-3,70$ & 203.20 & 1.744 \\
$2-3,100$ & 203.50 & 1.716 \\
$2-3,120$ & 203.70 & 1.698 \\
$2-3,120$ & 203.70 & 1.716 \\
$2-3,120$ & 203.70 & 1.774 \\
$2-3,130$ & 203.80 & 1.724 \\
$3-1,22$ & 361.22 & 1.731 \\
$3-1,74$ & 361.74 & 1.719 \\
$3-1,78$ & 361.78 & 1.701 \\
$3-6,30$ & 368.80 & 1.723 \\
$3-6,70$ & 369.20 & 1.672 \\
$3-6,100$ & 369.50 & 1.741 \\
$3-6,130$ & 369.80 & 1.754 \\
$4-5,10$ & 414.60 & 1.899 \\
$4-5,70$ & 415.20 & 1.884 \\
$4-5,100$ & 415.50 & 1.878 \\
$4-5,130$ & 415.80 & 1.890 \\
& &
\end{tabular}

Site 372

\begin{tabular}{lll}
$1-1,138$ & 113.38 & 1.658 \\
$1-1,145$ & 113.45 & 1.714 \\
$1-2,38$ & 113.88 & 1.656 \\
$1-2,72$ & 114.22 & 1.652 \\
$1-2,127$ & 114.77 & 1.704 \\
$1-3,25$ & 115.25 & 1.663 \\
$1-3,75$ & 115.75 & 1.656 \\
$1-3,115$ & 116.15 & 1.572 \\
$1-3,130$ & 116.30 & 1.562 \\
$2-2,118$ & 133.68 & 1.625 \\
$2-2,138$ & 133.88 & 1.693 \\
$2-3,10$ & 134.10 & 1.569 \\
$2-3,33$ & 134.33 & 1.684 \\
$2-3,64$ & 134.64 & 1.694 \\
$2-3,120$ & 135.20 & 1.766 \\
$2-3,135$ & 135.35 & 1.737 \\
$2-4,24$ & 135.74 & 1.628 \\
$2-4,73$ & 136.23 & 1.643 \\
$2-4,129$ & 136.79 & 1.708 \\
$3-1,58$ & 141.08 & 1.639 \\
$3-1,70$ & 141.20 & 1.641 \\
$3-1,134$ & 141.64 & 1.731 \\
$3-2,15$ & 142.15 & 1.605 \\
$3-2,72$ & 142.72 & 1.628 \\
$3-2,100$ & 143.00 & 1.701 \\
$3-2,138$ & 143.38 & 1.607 \\
$3-3,85$ & 144.35 & 1.641 \\
$3-3,122$ & 144.72 & 1.623 \\
$3-3,141$ & 144.91 & 1.654 \\
$4-1,68$ & 150.68 & 1.624 \\
\hline
\end{tabular}

TABLE 1 - Continued

\begin{tabular}{|c|c|c|}
\hline $\begin{array}{l}\text { Sample (Interval } \\
\text { in } \mathrm{cm} \text { ) }\end{array}$ & $\begin{array}{l}\text { Subbottom } \\
\text { Depth }(m)\end{array}$ & $\begin{array}{l}\text { Sonic Velocity } \\
(\mathrm{km} / \mathrm{sec})\end{array}$ \\
\hline $4-1,91$ & 150.91 & 1.657 \\
\hline $4-1,136$ & 151.36 & 1.561 \\
\hline $4-2,20$ & 151.70 & 1.648 \\
\hline $4-2,67$ & 152.17 & 1.638 \\
\hline $4-2,114$ & 152.64 & 1.653 \\
\hline $9-2,27$ & 199.27 & 1.696 \\
\hline $9-2,43$ & 199.43 & 1.693 \\
\hline $9-2,55$ & 199.55 & 1.683 \\
\hline $9-4,30$ & 202.30 & 1.678 \\
\hline $9-4,70$ & 202.70 & 1.660 \\
\hline $9-4,100$ & 203.00 & 1.703 \\
\hline $9-4,130$ & 203.30 & 1.696 \\
\hline $10-2,69$ & 209.19 & 1.647 \\
\hline $10-2,99$ & 209.49 & 1.669 \\
\hline $10-2,127$ & 209.77 & 1.676 \\
\hline $11-3,20$ & 219.70 & 1.669 \\
\hline $11-3,70$ & 220.20 & 1.632 \\
\hline $11-3,95$ & 220.45 & 1.638 \\
\hline $11-3,130$ & 220.80 & 1.595 \\
\hline $12-5,17$ & 232.17 & 1.673 \\
\hline $12-5,49$ & 232.49 & 1.645 \\
\hline $12-5,88$ & 232.88 & 1.636 \\
\hline $12-5,127$ & 233.27 & 1.667 \\
\hline $14-4,21$ & 249.98 & 1.633 \\
\hline $14-4,66$ & 250.43 & 1.684 \\
\hline $14-4,108$ & 250.85 & 1.670 \\
\hline $15-5,31$ & 260.81 & 1.705 \\
\hline $15-5,67$ & 261.17 & 1.688 \\
\hline $15-5,138$ & 261.88 & 1.666 \\
\hline $16-5,44$ & 270.44 & 1.654 \\
\hline $16-5,87$ & 270.87 & 1.725 \\
\hline $16-5,108$ & 271.08 & 1.693 \\
\hline $16-5,134$ & 271.34 & 1.700 \\
\hline $17-5,16$ & 279.66 & 1.743 \\
\hline $17-5,30$ & 279.80 & 1.757 \\
\hline $17-5,50$ & 280.00 & 1.759 \\
\hline $17-5,69$ & 280.19 & 1.788 \\
\hline $17-5,82$ & 280.32 & 1.756 \\
\hline $18-5,12$ & 289.12 & 1.739 \\
\hline $18-5,38$ & 289.38 & 1.736 \\
\hline $18-5,68$ & 289.68 & 1.756 \\
\hline $18-5,100$ & 290.00 & 1.748 \\
\hline $18-5,142$ & 290.42 & 1.750 \\
\hline $19-5,60$ & 299.10 & 1.683 \\
\hline $19-5,73$ & 299.23 & 1.717 \\
\hline $19-5,95$ & 299.45 & 1.834 \\
\hline $20-6,96$ & 310.87 & 1.706 \\
\hline $20-6,107$ & 310.98 & 1.771 \\
\hline $20-6,119$ & 311.10 & 1.731 \\
\hline $20-6,143$ & 311.34 & 1.834 \\
\hline $21-6,7$ & 319.07 & 1.713 \\
\hline $21-6,81$ & 319.81 & 1.745 \\
\hline $21-6,119$ & 320.19 & 1.732 \\
\hline $21-6,137$ & 320.37 & 1.812 \\
\hline $22-4,17$ & 325.67 & 1.756 \\
\hline $22-4,25$ & 325.75 & 1.737 \\
\hline $22-4,80$ & 326.30 & 1.794 \\
\hline
\end{tabular}


A. J. ERICKSON

TABLE 1 - Continued

\begin{tabular}{|c|c|c|}
\hline $\begin{array}{l}\text { Sample (Interval } \\
\text { in } \mathrm{cm} \text { ) }\end{array}$ & $\begin{array}{l}\text { Subbottom } \\
\text { Depth (m) }\end{array}$ & $\begin{array}{l}\text { Sonic Velocity } \\
(\mathrm{km} / \mathrm{sec})\end{array}$ \\
\hline $22-4,135$ & 326.85 & 1.657 \\
\hline $23-4,30$ & 335.30 & 1.709 \\
\hline $23-4,60$ & 335.60 & 1.763 \\
\hline $23-4,80$ & 335.80 & 1.738 \\
\hline $23-4,90$ & 335.90 & 1.739 \\
\hline $24-6,30$ & 348.22 & 1.792 \\
\hline $24-6,60$ & 348.52 & 1.824 \\
\hline $24-6,103$ & 348.95 & 1.799 \\
\hline $24-6,131$ & 349.23 & 1.780 \\
\hline $26-3,26$ & 362.26 & 1.808 \\
\hline $26-3,52$ & 362.52 & 1.762 \\
\hline $26-3,90$ & 362.90 & 1.838 \\
\hline $26-3,130$ & 363.30 & 1.791 \\
\hline $27-5,26$ & 374.76 . & 1.735 \\
\hline $27-5,63$ & 375.13 & 1.733 \\
\hline $27-5,105$ & 375.55 & 1.741 \\
\hline $27-5,138$ & 375.88 & 1.788 \\
\hline $28-5,10$ & 384.10 & 1.951 \\
\hline $28-5,66$ & 384.66 & 1.931 \\
\hline $28-5,105$ & 385.05 & 1.923 \\
\hline $28-5,145$ & 385.45 & 2.048 \\
\hline $29-4,12$ & 392.13 & 1.927 \\
\hline $29-4,33$ & 392.33 & 1.850 \\
\hline $29-4,72$ & 392.72 & 1.673 \\
\hline $29-4,95$ & 392.95 & 1.887 \\
\hline $30-5,12$ & 403.12 & 1.951 \\
\hline $30-5,47$ & 403.47 & 1.900 \\
\hline $30-5,86$ & 403.86 & 1.907 \\
\hline $30-5,129$ & 404.29 & 1.921 \\
\hline $31-5,8$ & 422.08 & 1.980 \\
\hline $31-5,23$ & 422.23 & 2.055 \\
\hline $31-5,58$ & 422.58 & 2.131 \\
\hline $31-5,86$ & 422.86 & 2.016 \\
\hline $31-5,110$ & 423.10 & 2.314 \\
\hline $31-5,111$ & 423.11 & 2.331 \\
\hline $32-6,12$ & 443.05 & 1.903 \\
\hline $32-6,65$ & 443.58 & 1.900 \\
\hline $32-6,96$ & 443.89 & 1.945 \\
\hline $32-6,116$ & 444.09 & 2.294 \\
\hline $32-6,134$ & 444.27 & 1.813 \\
\hline $32-6,136$ & 444.29 & 1.836 \\
\hline $33-1,140$ & 464.04 & 2.049 \\
\hline $33-1,19$ & 464.09 & 2.065 \\
\hline $33-1,19$ & 464.09 & 2.135 \\
\hline $33-1,51$ & 464.41 & 2.107 \\
\hline $33-1,98$ & 464.88 & 2.222 \\
\hline $33-6,11$ & 471.51 & 1.859 \\
\hline $33-6,31$ & 471.71 & 1.956 \\
\hline $33-6,61$ & 472.01 & 1.823 \\
\hline $33-6,139$ & 472.79 & 1.871 \\
\hline
\end{tabular}

Site 374

\begin{tabular}{lll}
$2-2,30$ & 158.80 & 1.605 \\
$2-2,80$ & 159.30 & 1.643 \\
$2-2,100$ & 159.50 & 1.616 \\
$2-2,135$ & 159.85 & 1.613 \\
$3-1,85$ & 208.85 & 1.621 \\
$3-1,116$ & 209.16 & 1.641 \\
$3-1,132$ & 209.32 & 1.697 \\
$3-1,147$ & 209.47 & 1.653 \\
$4-3,30$ & 254.80 & 1.685 \\
$4-3,70$ & 255.20 & 1.741 \\
$4-3,100$ & 255.50 & 1.743 \\
$4-3,137$ & 255.87 & 1.749 \\
$5-2,22$ & 298.72 & 1.806 \\
$5-2,53$ & 299.03 & 1.793 \\
$5-2,75$ & 299.25 & 1.794 \\
\hline
\end{tabular}

TABLE 1 - Continued

\begin{tabular}{|c|c|c|}
\hline $\begin{array}{l}\text { Sample (Interval } \\
\text { in } \mathrm{cm} \text { ) }\end{array}$ & $\begin{array}{c}\text { Subbottom } \\
\text { Depth (m) }\end{array}$ & $\begin{array}{l}\text { Sonic Velocity } \\
(\mathrm{km} / \mathrm{sec})\end{array}$ \\
\hline $5-2,112$ & 299.62 & 1.851 \\
\hline $5-2,142$ & 299.92 & 1.856 \\
\hline $5-3,22$ & 300.22 & 1.807 \\
\hline $5-3,40$ & 300.40 & 1.849 \\
\hline $5-3,79$ & 300.79 & 1.839 \\
\hline $5-3,113$ & 301.13 & 1.869 \\
\hline $5-3,139$ & 301.39 & 1.840 \\
\hline $5-4,28$ & 301.78 & 1.882 \\
\hline $5-4,47$ & 301.97 & 1.845 \\
\hline $5-4,72$ & 302.22 & 1.846 \\
\hline $5-4,104$ & 302.54 & 1.921 \\
\hline $5-4,127$ & 302.77 & 1.876 \\
\hline $5-5,20$ & 303.20 & 1.890 \\
\hline $5-5,45$ & 303.45 & 1.911 \\
\hline $5-5,80$ & 303.80 & 1.894 \\
\hline $5-5,109$ & 304.09 & 1.866 \\
\hline $6-4,26$ & 335.74 & 1.870 \\
\hline $6-4,60$ & 336.08 & 1.892 \\
\hline $6-4,114$ & 336.62 & 1.885 \\
\hline $6-4,138$ & 336.86 & 1.872 \\
\hline $6-5,32$ & 337.30 & 1.807 \\
\hline $6-5,54$ & 337.52 & 1.899 \\
\hline $6-5,95$ & 337.93 & 1.849 \\
\hline $6-5,127$ & 338.25 & 1.854 \\
\hline $6-6,33$ & 338.81 & 1.775 \\
\hline $6-6,55$ & 339.03 & 1.786 \\
\hline $6-6,112$ & 339.60 & 1.820 \\
\hline $6-6,141$ & 339.89 & 1.838 \\
\hline $7-4,12$ & 345.02 & 1.948 \\
\hline $7-4,43$ & 345.33 & 1.877 \\
\hline $7-4,72$ & 345.62 & 1.875 \\
\hline $7-4,108$ & 345.98 & 1.876 \\
\hline $7-4,129$ & 346.19 & 1.917 \\
\hline $7-5,30$ & 346.70 & 1.911 \\
\hline $7-5,36$ & 346.76 & 1.905 \\
\hline $7-5,70$ & 347.10 & 1.917 \\
\hline $7-5,102$ & 347.42 & 1.914 \\
\hline $7-5,129$ & 347.69 & 1.969 \\
\hline $7-6,11$ & 348.01 & 1.864 \\
\hline $7-6,36$ & 348.26 & 1.867 \\
\hline $7-6,73$ & 348.63 & 1.927 \\
\hline $7-6,101$ & 348.91 & 1.845 \\
\hline $7-6,117$ & 349.07 & 1.813 \\
\hline $8-3,20$ & 352.70 & 1.875 \\
\hline $8-3,44$ & 352.94 & 1.935 \\
\hline $8-3,78$ & 353.28 & 1.931 \\
\hline $8-3,121$ & 353.71 & 1.950 \\
\hline $8-3,140$ & 353.90 & 1.993 \\
\hline $8-4,61$ & 354.61 & 1.853 \\
\hline $8-4,61$ & 354.61 & 1.925 \\
\hline $8-4,90$ & 354.90 & 2.041 \\
\hline $8-4,107$ & 355.07 & 2.011 \\
\hline $8-4,139$ & 355.39 & 1.928 \\
\hline $9-4,26$ & 363.76 & 1.934 \\
\hline $9-4,40$ & 363.90 & 1.985 \\
\hline $9-4,70$ & 364.20 & 2.000 \\
\hline $10-1,108$ & 369.58 & 2.056 \\
\hline $10-1,118$ & 369.68 & 2.058 \\
\hline $12-2,9$ & 383.09 & 1.609 \\
\hline $12-2,24$ & 383.24 & 1.636 \\
\hline $12-2,40$ & 383.40 & 1.890 \\
\hline $12-2,51$ & 383.61 & 1.849 \\
\hline $12-2,66$ & 383.66 & 1.920 \\
\hline $13-2,17$ & 389.17 & 1.968 \\
\hline $13-2,43$ & 389.43 & 1.953 \\
\hline $13-2,62$ & 389.62 & 1.930 \\
\hline $13-2,72$ & 389.72 & 1.956 \\
\hline $14-2,20$ & 394.20 & 1.976 \\
\hline
\end{tabular}


TABLE 1 - Continued

\begin{tabular}{lcr}
\hline $\begin{array}{c}\text { Sample (Interval } \\
\text { in cm) }\end{array}$ & $\begin{array}{c}\text { Subbottom } \\
\text { Depth }(\mathrm{m})\end{array}$ & $\begin{array}{r}\text { Sonic Velocity } \\
(\mathrm{km} / \mathrm{sec})\end{array}$ \\
\hline & & \\
$14-2,50$ & 394.50 & 2.002 \\
$14-2,75$ & 394.75 & 2.011 \\
$14-2,100$ & 395.00 & 1.976 \\
$14-2,123$ & 395.23 & 1.917 \\
$14-2,140$ & 395.40 & 2.003 \\
$15-2,30$ & 398.80 & 2.626 \\
$15-2,57$ & 399.07 & 2.665 \\
$15-2,61$ & 399.11 & 2.716 \\
& & \\
Site 375 & & \\
& & \\
$7-4,6$ & 569.96 & 1.984 \\
$7-4,42$ & 570.32 & 2.016 \\
$7-4,80$ & 570.70 & 2.011 \\
$7-4,115$ & 571.05 & 1.981 \\
$7-4,146$ & 571.36 & 1.997 \\
$9-3,14$ & 654.08 & 2.786 \\
$9-3,24$ & 654.18 & 2.692 \\
$9-3,74$ & 654.68 & 2.565 \\
$9-3,87$ & 654.81 & 2.704 \\
$9-3,102$ & 654.96 & 2.598
\end{tabular}

Site 376

\begin{tabular}{lrl}
$1-2,23$ & 1.73 & 1.517 \\
$1-2,56$ & 2.06 & 1.532 \\
$1-2,85$ & 2.35 & 1.527 \\
$1-2,106$ & 2.56 & 1.525 \\
$1-2,132$ & 2.82 & 1.526 \\
$1-4,35$ & 4.85 & 1.508 \\
$1-4,60$ & 5.10 & 1.511 \\
$1-4,82$ & 5.32 & 1.498 \\
$1-4,102$ & 5.52 & 1.523 \\
$1-4,135$ & 5.85 & 1.536 \\
$2-2,18$ & 9.18 & 1.524 \\
$2-2,48$ & 9.48 & 1.529 \\
$2-2,62$ & 9.62 & 1.602 \\
$2-2,71$ & 9.71 & 1.531 \\
$2-2,100$ & 10.00 & 1.519 \\
$2-2,135$ & 10.35 & 1.544 \\
$2-3,22$ & 10.72 & 1.494 \\
$2-3,42$ & 10.92 & 1.542 \\
$2-3,73$ & 11.23 & 1.549 \\
$2-3,100$ & 11.50 & 1.533 \\
$2-3,135$ & 11.85 & 1.567 \\
$3-3,6$ & 20.06 & 1.548 \\
$3-3,13$ & 20.40 & 1.580 \\
$3-3,38$ & 20.86 & 1.568 \\
$3-3,104$ & 21.04 & 1.585 \\
$3-3,144$ & 21.44 & 1.573 \\
$3-4,27$ & 21.77 & 1.547 \\
$3-4,70$ & 22.20 & 1.564 \\
$3-4,89$ & 22.39 & 1.538 \\
$3-4,106$ & 22.56 & 1.534 \\
$3-4,142$ & 22.92 & 1.592 \\
$4-2,25$ & 28.25 & 1.527 \\
$4-2,60$ & 28.60 & 1.540 \\
$4-2,98$ & 28.98 & 1.537 \\
$4-2,140$ & 29.40 & 1.533 \\
$5-2,20$ & 37.70 & 1.557 \\
$5-2,44$ & 37.94 & 1.573 \\
$5-2,73$ & 38.23 & 1.543 \\
$5-2,95$ & 38.45 & 1.586 \\
$5-2,114$ & 38.64 & 1.564 \\
$5-2,141$ & 38.91 & 1.571 \\
$5-5,18$ & 42.18 & 1.590 \\
$5-5,31$ & 42.31 & 1.592 \\
$5-5,50$ & 42.50 & 1.609 \\
\hline
\end{tabular}

TABLE 1 - Continued

\begin{tabular}{|c|c|c|}
\hline $\begin{array}{l}\text { Sample (Interval } \\
\text { in } \mathrm{cm} \text { ) }\end{array}$ & $\begin{array}{c}\text { Subbottom } \\
\text { Depth (m) }\end{array}$ & $\begin{array}{l}\text { Sonic Velocity } \\
(\mathrm{km} / \mathrm{sec})\end{array}$ \\
\hline $5-5,73$ & 42.73 & 1.592 \\
\hline $5-5,96$ & 42.96 & 1.580 \\
\hline $5-5,180$ & 43.30 & 1.582 \\
\hline $6-3,48$ & 48.98 & 1.584 \\
\hline $6-3,70$ & 49.20 & 1.584 \\
\hline $6-3,79$ & 49.29 & 1.576 \\
\hline $6-3,96$ & 49.46 & 1.569 \\
\hline $6-3,113$ & 49.63 & 1.583 \\
\hline $6-3,137$ & 49.87 & 1.597 \\
\hline $6-4,10$ & 50.10 & 1.636 \\
\hline $6-4,29$ & 50.29 & 1.602 \\
\hline $6-4,68$ & 50.68 & 1.612 \\
\hline $6-4,86$ & 50.86 & 1.580 \\
\hline $6-4,90$ & 50.90 & 1.568 \\
\hline $6-4,106$ & 51.06 & 1.661 \\
\hline $6-4,140$ & 51.40 & 1.594 \\
\hline $7-2,6$ & 56.56 & 1.585 \\
\hline $7-2,25$ & 56.75 & 1.628 \\
\hline $7-2,48$ & 56.98 & 1.577 \\
\hline $7-2,72$ & 57.22 & 1.594 \\
\hline $7-2,106$ & 57.56 & 1.583 \\
\hline $7-2,146$ & 57.96 & 1.573 \\
\hline $8-2,8$ & 66.08 & 1.586 \\
\hline $8-2,33$ & 66.33 & 1.592 \\
\hline $8-2,64$ & 66.64 & 1.608 \\
\hline $8-2,103$ & 67.03 & 1.735 \\
\hline $8-2,120$ & 67.20 & 1.706 \\
\hline $8-2,140$ & 67.40 & 1.652 \\
\hline $9-2,20$ & 75.70 & 1.579 \\
\hline $9-2,43$ & 75.93 & 1.596 \\
\hline $9-2,54$ & 76.04 & 1.594 \\
\hline $9-2,81$ & 76.31 & 1.585 \\
\hline $9-2,88$ & 76.38 & 1.792 \\
\hline $9-2,117$ & 76.67 & 1.577 \\
\hline $9-3,18$ & 77.18 & 1.596 \\
\hline $9-3,49$ & 77.49 & 1.633 \\
\hline $9-3,77$ & 77.77 & 1.684 \\
\hline $9-3,104$ & 78.04 & 1.631 \\
\hline $9-3,123$ & 78.23 & 1.674 \\
\hline $9-3,138$ & 78.38 & 1.659 \\
\hline $10-2,9$ & 85.09 & 1.642 \\
\hline $10-2,18$ & 85.18 & 1.694 \\
\hline $10-2,57$ & 85.57 & 1.597 \\
\hline $10-2,77$ & 85.77 & 1.659 \\
\hline $10-2,120$ & 86.20 & 1.706 \\
\hline $10-2,146$ & 86.46 & 1.695 \\
\hline $11-3,20$ & 96.20 & 1.612 \\
\hline $11-3,40$ & 96.40 & 1.792 \\
\hline $11-3,75$ & 96.75 & 1.783 \\
\hline $11-3,109$ & 97.09 & 1.801 \\
\hline $11-3,148$ & 97.48 & 1.633 \\
\hline $12-2,6$ & 104.06 & 1.723 \\
\hline $12-2,58$ & 104.58 & 1.665 \\
\hline $12-2,71$ & 104.71 & 1.674 \\
\hline $12-2,103$ & 105.03 & 1.668 \\
\hline $12-2,145$ & 105.45 & 1.698 \\
\hline $12-4,18$ & 107.18 & 1.655 \\
\hline $12-4,44$ & 107.44 & 1.700 \\
\hline $12-4,60$ & 107.50 & 1.675 \\
\hline $12-4,78$ & 107.78 & 1.711 \\
\hline $12-4,119$ & 108.19 & 1.713 \\
\hline $12-4,142$ & 108.42 & 1.704 \\
\hline $13-2,5$ & 113.55 & 1.627 \\
\hline $13-2,22$ & 113.72 & 1.660 \\
\hline $13-2,48$ & 113.98 & 1.697 \\
\hline $13-2,80$ & 114.30 & 1.691 \\
\hline $13-2,112$ & 114.62 & 1.703 \\
\hline $13-2,137$ & 114.87 & 1.744 \\
\hline
\end{tabular}


TABLE 1 - Continued

\begin{tabular}{lcc}
\hline $\begin{array}{c}\text { Sample (Interval } \\
\text { in cm) }\end{array}$ & $\begin{array}{c}\text { Subbottom } \\
\text { Depth }(\mathrm{m})\end{array}$ & $\begin{array}{c}\text { Sonic Velocity } \\
(\mathrm{km} / \mathrm{sec})\end{array}$ \\
\hline & & \\
$13-4,20$ & 116.70 & 1.646 \\
$13-4,56$ & 117.06 & 1.677 \\
$13-4,89$ & 117.39 & 1.674 \\
$13-4,106$ & 117.56 & 1.657 \\
$13-4,122$ & 117.72 & 1.727 \\
$13-4,140$ & 117.90 & 1.660 \\
$15-3,11$ & 134.11 & 1.763 \\
$15-3,47$ & 134.47 & 1.751 \\
$15-3,94$ & 134.94 & 1.839 \\
$15-3,141$ & 135.41 & 1.693 \\
$16-1,40$ & 140.90 & 1.711 \\
$16-1,65$ & 141.15 & 1.723 \\
$16-1,74$ & 141.24 & 1.677 \\
$19-1,148$ & 170.48 & 4.455 \\
$19-1,148$ & 170.48 & 4.494 \\
$19-1,148$ & 170.48 & 4.713 \\
& &
\end{tabular}

Hole 378

$\begin{array}{lrl}1-1,110 & 85.10 & 1.528 \\ 1-1,112 & 85.12 & 1.531 \\ 1-1,138 & 85.38 & 1.550 \\ 1-1,146 & 85.46 & 1.525 \\ 2-5,6 & 99.56 & 1.546 \\ 2-5,40 & 99.90 & 1.561 \\ 2-5,77 & 100.27 & 1.587 \\ 2-5,127 & 100.77 & 1.559 \\ 2-5,145 & 100.95 & 1.559 \\ 3-3,16 & 106.16 & 1.541 \\ 3-3,59 & 106.59 & 1.554 \\ 3-3,78 & 106.78 & 1.550 \\ 3-3,98 & 106.98 & 1.614 \\ 3-3,130 & 107.30 & 1.582 \\ 5-2,6 & 123.56 & 1.503 \\ 5-2,26 & 123.76 & 1.519 \\ 5-2,104 & 124.54 & 1.518 \\ 5-2,118 & 124.68 & 1.548 \\ 5-3,14 & 144.14 & 1.568 \\ 6-3,44 & 144.44 & 1.579 \\ 6-3,89 & 144.89 & 1.583 \\ 6-3,113 & 145.13 & 1.576 \\ 6-3,139 & 145.39 & 1.629 \\ 7-3,16 & 172.86 & 1.648 \\ 7-3,45 & 172.95 & 1.616 \\ 7-3,89 & 173.39 & 1.642 \\ 7-3,100 & 173.50 & 1.680 \\ 8-2,16 & 218.66 & 1.620 \\ 8-2,39 & 218.89 & 1.602 \\ 8-2,83 & 219.33 & 1.657 \\ 8-2,112 & 219.62 & 1.686 \\ 11-3,10 & 305.60 & 1.626 \\ 11-3,40 & 305.90 & 1.597 \\ 11-3,65 & 306.15 & 1.611 \\ 11-3,93 & 306.43 & 1.655 \\ 11-3,135 & 306.85 & 1.616\end{array}$

Hole 378A

$\begin{array}{lll}1-5,10 & 52.10 & 1.529 \\ 1-5,39 & 52.39 & 1.505 \\ 1-5,73 & 52.73 & 1.526 \\ 1-5,118 & 53.18 & 1.542\end{array}$

TABLE 2

\begin{tabular}{cccc}
\hline & Horizontal & Vertical \\
Sample (Interval & Subbottom & Velocity & Velocity \\
in $\mathrm{cm})$ & Depth $(\mathrm{m})$ & $(\mathrm{km} / \mathrm{sec})$ & $(\mathrm{km} / \mathrm{sec})$ \\
\hline
\end{tabular}

Site 371

$\begin{array}{lllll}4-5,75 & 415.25 & 1.868 & (1) & 1.839\end{array}$

Site 372

$\begin{array}{ll}31-1,139 & 417.39 \\ 31-6,25 & 423.75 \\ 32-4 & 439.93 \\ 32-4,23 & 440.16 \\ 32-5,118 & 442.59 \\ 33-1 & 467.00 \\ 33-4,74 & 469.12 \\ 34-2,22 & 493.70 \\ 34-3,144 & 496.43 \\ 35-2,7 & 530.57 \\ 35-2,68 & 531.18 \\ 35-2,130 & 531.80 \\ 35-3,5 & 532.03 \\ 36-4,134 & 574.26 \\ 37-2,39 & 607.87 \\ 38-1,137 & 645.72 \\ 38-5,12 & 650.47 \\ 39-1,9 & 682.47 \\ 39-3,129 & 686.67 \\ 40-2,78 & 722.28 \\ 40-3,2 & 723.01 \\ 41-4,16 & 762.94 \\ 41-4,141 & 764.18 \\ 41-6,86 & 766.63 \\ 42-1,117 & 788.01 \\ 42-2,71 & 798.19 \\ 44-1,119 & 835.58 \\ 45-3,143 & 876.84 \\ 46-3,9 & 884.57\end{array}$

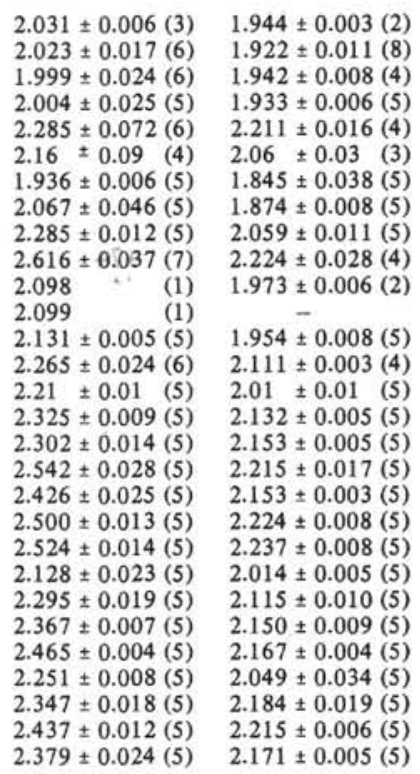

Hole 373A

$7-3,22$

$7-4,20$

$7-4,118$

365.70

367.18

368.16

$7-4,147$

368.45

$5.065 \pm 0.022(5)$

$5.020 \pm 0.019$ (5) $\quad 5.150 \pm 0.013$ (5) $4.399 \pm 0.021(5) \quad 4.443 \pm 0.010(5)$ $4.106 \pm 0.172(5) \quad 4.303 \pm 0.065(5)$

Site 374

$\begin{array}{ll}16-1,96 & 407.44 \\ 16-1,119 & 407.67 \\ 17-1,22 & 411.22 \\ 17-1,35 & 411.35 \\ 19-1,90 & 418.88 \\ 20-1,113 & 421.11 \\ 22-1,38 & 435.38\end{array}$

$\begin{array}{lc}4.732 \pm 0.117(2) & 4.586 \pm 0.110(2) \\ 4.754 \pm 0.316(2) & 4.592 \pm 0.087(2) \\ 4.839 \pm 0.046(3) & 4.730 \pm 0.036(3) \\ 4.783 \pm 0.203(4) & - \\ 5.091 \pm 0.424(5) & 4.672 \pm 0.079(5) \\ 5.028 \pm 0.027(5) & 4.933 \pm 0.020(5) \\ 4.888 \pm 0.145(4) & 4.983 \pm 0.093(4)\end{array}$

Site 375

$\begin{array}{ll}1-1,146 & 138.96 \\ 2-4,40 & 194.38 \\ 4-4,5 & 250.05 \\ 10-2,71 & 677.70 \\ 11-1,32 & 733.32 \\ 11-2,133 & 735.81 \\ 11-2,142 & 735.91\end{array}$

$4.576 \pm 0.079(3) \quad 4.687 \pm 0.088(4)$ $4.866 \pm 0.018(5) \quad 4.539 \pm 0.048(5)$ $2.005 \pm 0.015(5) \quad 1.931 \pm 0.013(5)$ $2.635 \pm 0.035(5) \quad 2.495 \pm 0.018(5)$ $5.562 \pm 0.129(5) \quad 5.526 \pm 0.153(5)$ $5.565 \pm 0.053(10)$

$2.851 \pm 0.077$ (5) $\quad 2.594 \pm 0.024$ (5)

Site 376

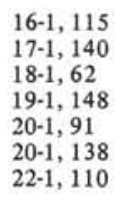

141.65

151.40

160.12

170.48

185.90

186.38

206.06

$4.423 \pm 0.060$ (3) $\quad 4.431 \pm 0.054$ (3) $4.624 \pm 0.019(3) \quad 4.603 \pm 0.009(3)$ $4.651 \pm 0.043(5) \quad 4.588 \pm 0.138(5)$ $4.554 \pm 0.139(3)$

$4.524 \pm 0.020(5) \quad 4.526 \pm 0.058(5)$

$3.641 \pm 0.109$ (4) $\quad 3.602 \pm 0.017$ (4)

$5.165 \pm 0.095(5) \quad-$

Hole 378A

$\begin{array}{llll}4-1,69 & 303.19 & 4.670 \pm 0.071(5) & 5.821 \pm 0.062(5) \\ 5-1,115 & 321.14 & 4.805 \pm 0.032(5) & 6.026 \pm 0.100(5) \\ 6-1,22 & 330.20 & 4.189 \pm 0.024(5) & 4.914 \pm 0.016(5)\end{array}$


PHYSICAL PROPERTIES DATA

TABLE 3

\begin{tabular}{lrrr}
\hline Core - Section & $\begin{array}{c}\text { Subbottom } \\
\text { Depth (m) }\end{array}$ & $\begin{array}{c}\text { Wet Bulk } \\
\text { Density }(\mathrm{g} / \mathrm{cc})\end{array}$ & $\begin{array}{r}\text { Porosity } \\
\text { (wt. 90) }\end{array}$ \\
\hline & & & \\
Site 371 & & & \\
& & & \\
$1-3$ & 4.42 & 1.730 & 56.63 \\
$1-4$ & 4.72 & 1.817 & 51.26 \\
$1-5$ & 6.68 & 1.746 & 55.65 \\
$1-6$ & 8.63 & 1.764 & 54.52 \\
$2-2$ & 201.68 & 1.849 & 49.26 \\
$2-3$ & 203.18 & 1.971 & 41.80 \\
$3-2$ & 363.78 & 1.978 & 41.35 \\
$3-4$ & 366.18 & 2.035 & 37.86 \\
$3-5$ & 367.38 & 2.019 & 38.80 \\
$4-2$ & 410.83 & 2.015 & 39.09 \\
$4-4$ & 413.38 & 2.081 & 35.03 \\
$4-6$ & 417.28 & 2.128 & 32.14 \\
$5-2$ & 468.88 & 2.070 & 35.69 \\
$5-4$ & 471.43 & 2.103 & 33.64 \\
$5-6$ & 474.28 & 2.147 & 30.98 \\
$8-1$ & 547.42 & 2.568 & 5.06 \\
$8-2$ & 547.57 & 2.524 & 7.76
\end{tabular}

Site 372

\begin{tabular}{|c|c|c|c|}
\hline $1-2$ & 114.48 & 1.808 & 51.83 \\
\hline $1-3$ & 115.38 & 1.874 & 47.74 \\
\hline $2-2$ & 133.78 & 1.935 & 44.00 \\
\hline $2-4$ & 135.58 & 1.990 & 40.62 \\
\hline $3-2$ & 142.53 & 1.900 & 46.18 \\
\hline $3-3$ & 144.78 & 1.943 & 43.49 \\
\hline $4-2$ & 152.78 & 1.966 & 42.11 \\
\hline $9-2$ & 199.38 & 2.030 & 38.15 \\
\hline $9-3$ & 201.03 & 1.942 & 43.59 \\
\hline $9-3$ & 201.48 & 1.956 & 42.70 \\
\hline $9-4$ & 202.83 & 2.101 & 33.79 \\
\hline $10-2$ & 209.63 & 1.887 & 46.95 \\
\hline $11-3$ & 219.58 & 1.897 & 46.33 \\
\hline $12-5$ & 232.53 & 1.966 & 42.11 \\
\hline $13-5$ & 242.72 & 1.932 & 44.18 \\
\hline $14-4$ & 249.85 & 1.939 & 43.75 \\
\hline $15-5$ & 261.93 & 1.846 & 49.49 \\
\hline $16-5$ & 270.38 & 1.914 & 45.29 \\
\hline $17-5$ & 280.18 & 1.846 & 49.47 \\
\hline $18-5$ & 290.43 & 1.862 & 48.49 \\
\hline $19-5$ & 298.58 & 1.812 & 51.59 \\
\hline $20-6$ & 311.04 & 2.055 & 36.59 \\
\hline $21-6$ & 319.53 & 1.953 & 42.87 \\
\hline $22-4$ & 326.33 & 1.871 & 47.91 \\
\hline $23-4$ & 335.23 & 1.883 & 47.20 \\
\hline $24-6$ & 348.75 & 2.069 & 35.73 \\
\hline $25-6$ & 358.39 & 1.980 & 41.23 \\
\hline $26-3$ & 362.83 & 2.009 & 39.46 \\
\hline $31-4$ & 421.63 & 2.146 & 31.01 \\
\hline $31-6$ & 424.18 & 2.019 & 38.82 \\
\hline $32-4$ & 440.16 & 2.116 & 32.84 \\
\hline $32-5$ & 442.71 & 2.131 & 31.94 \\
\hline $33-4$ & 469.23 & 2.056 & 36.58 \\
\hline $34-2$ & 493.58 & 2.051 & 36.88 \\
\hline $34-3$ & 495.23 & 1.791 & 54.95 \\
\hline $36-3$ & 571.64 & 2.061 & 36.23 \\
\hline $37-2$ & 608.02 & 2.062 & 36.21 \\
\hline $38-1$ & 645.19 & 2.074 & 35.45 \\
\hline $38-5$ & 651.64 & 2.168 & 29.64 \\
\hline $39-1$ & 683.37 & 2.190 & 28.30 \\
\hline $39-3$ & 686.22 & 2.103 & 33.64 \\
\hline $40-2$ & 721.72 & 2.128 & 32.14 \\
\hline $40-3$ & 723.97 & 2.145 & 31.07 \\
\hline $41-4$ & 764.21 & 2.269 & 23.46 \\
\hline $41-6$ & 766.16 & 2.250 & 24.62 \\
\hline $42-1$ & 788.28 & 2.134 & 31.77 \\
\hline
\end{tabular}

TABLE 3 - Continued

\begin{tabular}{crcr}
\hline Core - Section & $\begin{array}{c}\text { Subbottom } \\
\text { Depth (m) }\end{array}$ & $\begin{array}{c}\text { Wet Bulk } \\
\text { Density (g/cc) }\end{array}$ & $\begin{array}{c}\text { Porosity } \\
\text { (wt. 90) }\end{array}$ \\
\hline & & & \\
$43-2$ & 798.32 & 2.228 & 25.97 \\
$44-1$ & 835.22 & 2.248 & 24.71 \\
$45-3$ & 876.54 & 2.243 & 25.02 \\
$46-3$ & 885.92 & 2.362 & 17.72
\end{tabular}

Site 374

$\begin{array}{llll}1-2 & 102.23 & 1.816 & 51.30 \\ 2-3 & 161.13 & 1.879 & 47.47 \\ 4-2 & 254.43 & 1.858 & 48.73 \\ 4-3 & 255.18 & 1.985 & 40.92 \\ 4-4 & 256.53 & 1.883 & 47.19 \\ 5-3 & 300.68 & 1.954 & 42.84 \\ 5-4 & 301.58 & 1.862 & 48.50 \\ 5-5 & 303.83 & 1.865 & 48.32 \\ 6-4 & 335.56 & 1.918 & 45.06 \\ 6-5 & 337.06 & 1.928 & 44.46 \\ 7-1 & 340.93 & 1.940 & 43.68 \\ 7-2 & 343.03 & 1.956 & 42.72 \\ 7-3 & 344.08 & 1.964 & 42.19 \\ 7-4 & 345.73 & 1.966 & 42.11 \\ 7-5 & 347.08 & 1.968 & 41.98 \\ 7-6 & 349.18 & 2.085 & 34.76 \\ 8-2 & 351.68 & 1.994 & 40.36 \\ 8-3 & 353.33 & 1.989 & 40.70 \\ 8-4 & 354.98 & 2.027 & 38.37 \\ 9-1 & 360.28 & 1.984 & 40.98 \\ 9-2 & 360.73 & 1.986 & 40.86 \\ 9-3 & 362.98 & 1.964 & 42.24 \\ 9-4 & 363.73 & 1.942 & 43.55 \\ 11-1 & 378.08 & 1.963 & 42.25 \\ 11-2 & 380.93 & 1.909 & 45.58 \\ 12-1 & 382.48 & 1.981 & 41.18 \\ 12-2 & 383.08 & 1.971 & 41.78 \\ 13-2 & 389.38 & 2.082 & 34.97 \\ 13-3 & 391.78 & 2.057 & 36.48 \\ 14-1 & 392.88 & 2.018 & 38.89 \\ 14-2 & 394.98 & 2.025 & 38.47 \\ 15-1 & 398.13 & 2.019 & 38.86 \\ 15-2 & 398.73 & 2.038 & 37.68 \\ 16-1 & 407.33 & 2.292 & 22.05 \\ 17-1 & 411.38 & 2.264 & 23.75 \\ 18-1 & 417.13 & 2.271 & 23.34 \\ 19-1 & 418.83 & 2.252 & 24.49 \\ 20-1 & 420.83 & 2.350 & 18.49 \\ 22-1 & 435.38 & 2.469 & 11.12\end{array}$

Site 375

$\begin{array}{llll}6-3 & 464.23 & 1.936 & 43.91 \\ 6-4 & 466.03 & 2.142 & 31.24 \\ 6-6 & 469.78 & 2.041 & 37.45 \\ 7-5 & 572.52 & 2.149 & 30.84 \\ 9-3 & 654.16 & 2.314 & 20.70 \\ 10-2 & 677.37 & 2.310 & 20.90 \\ 11-2 & 734.87 & 2.391 & 15.92\end{array}$

Site 376

\begin{tabular}{rrrr}
$1-2$ & 1.72 & 1.677 & 59.86 \\
$1-4$ & 5.62 & 1.744 & 55.75 \\
$2-2$ & 9.98 & 1.704 & 58.19 \\
$2-3$ & 11.03 & 1.799 & 52.39 \\
$3-3$ & 20.98 & 1.769 & 54.23 \\
$3-4$ & 21.88 & 1.769 & 54.20 \\
$4-2$ & 28.83 & 1.761 & 54.70 \\
$5-2$ & 38.93 & 1.762 & 54.67 \\
$5-5$ & 43.13 & 1.801 & 52.23 \\
\hline
\end{tabular}


TABLE 3 - Continued

\begin{tabular}{lrcc}
\hline Core-Section & $\begin{array}{c}\text { Subbottom } \\
\text { Depth }(\mathrm{m})\end{array}$ & $\begin{array}{c}\text { Wet Bulk } \\
\text { Density }(\mathrm{g} / \mathrm{cc})\end{array}$ & $\begin{array}{c}\text { Porosity } \\
\text { (wt. 90) }\end{array}$ \\
\hline & & & \\
$6-3$ & 49.93 & 1.846 & 49.45 \\
$6-4$ & 51.13 & 1.943 & 43.53 \\
$7-2$ & 57.78 & 1.913 & 45.33 \\
$8-2$ & 67.13 & 1.961 & 42.41 \\
$9-2$ & 76.33 & 1.813 & 51.54 \\
$9-3$ & 78.43 & 1.848 & 49.33 \\
$10-2$ & 86.43 & 2.003 & 39.81 \\
$11-3$ & 96.68 & 1.990 & 40.61 \\
$11-3$ & 96.68 & 1.990 & 40.61 \\
$12-2$ & 105.43 & 1.897 & 46.35 \\
$12-4$ & 108.43 & 1.945 & 43.40 \\
$13-2$ & 114.93 & 1.946 & 43.30 \\
$13-4$ & 117.18 & 1.987 & 40.83 \\
$15-3$ & 134.83 & 2.083 & 34.92
\end{tabular}

Site 377

$\begin{array}{llll}1-2 & 192.38 & 1.918 & 45.04\end{array}$

Hole 378

$\begin{array}{lrrr}1-1 & 85.43 & 1.678 & 59.83 \\ 1-2 & 86.53 & 1.719 & 57.32 \\ 2-2 & 95.98 & 1.704 & 58.19 \\ 2-5 & 100.63 & 1.858 & 48.76 \\ 3-2 & 105.93 & 1.819 & 51.13 \\ 5-2 & 124.78 & 1.660 & 60.94 \\ 6-3 & 145.28 & 1.812 & 51.55 \\ 7-2 & 171.53 & 1.776 & 53.80 \\ 7-3 & 173.48 & 1.738 & 56.15 \\ 8-2 & 219.63 & 1.614 & 63.75 \\ 11-3 & 306.63 & 1.909 & 45.60 \\ 11-4 & 307.98 & 1.897 & 46.35\end{array}$

Hole 378A

\begin{tabular}{llll}
$3-5$ & 299.80 & 1.931 & 44.25 \\
\hline
\end{tabular}

TABLE 4

\begin{tabular}{ccccc}
\hline $\begin{array}{c}\text { Sample } \\
\text { Interval in } \mathrm{cm})\end{array}$ & $\begin{array}{c}\text { Subbottom } \\
\text { Depth }(\mathrm{m})\end{array}$ & $\begin{array}{c}\text { Density } \\
(\mathrm{g} / \mathrm{cc})\end{array}$ & $\begin{array}{c}\text { Water Content } \\
(\%)\end{array}$ & $\begin{array}{c}\text { Porosity } \\
(\%)\end{array}$ \\
\hline
\end{tabular}

\section{Site 371}

$\begin{array}{lrlll}1-2,57 & 2.08 & 1.556 & 39.75 & 61.85 \\ 1-5,57 & 6.58 & 1.629 & 34.12 & 55.57 \\ 2-2,57 & 201.58 & 1.820 & 25.56 & 46.53 \\ 2-3,57 & 203.08 & 1.792 & 22.45 & 40.23 \\ 3-3,57 & 364.58 & 1.923 & 21.07 & 40.52 \\ 3-5,55 & 367.56 & 1.895 & 22.60 & 42.83 \\ 4-2,57 & 410.58 & 1.962 & 17.28 & 33.90 \\ 4-6,56 & 416.57 & 1.916 & 17.59 & 33.70 \\ 5-2,57 & 467.93 & 2.000 & 18.32 & 36.64 \\ 5-6,57 & 473.93 & 2.217 & 15.14 & 33.57 \\ 8-2,108 & 548.59 & 2.485 & 14.25 & 35.40\end{array}$

Site 372

\begin{tabular}{lllll}
$1-1,33$ & 112.34 & 1.528 & 29.07 & 44.40 \\
$1-2,57$ & 114.08 & 1.860 & 29.45 & 54.77 \\
$1-3,57$ & 115.58 & 1.785 & 32.62 & 58.23 \\
$1-4,46$ & 116.97 & 1.639 & 27.88 & 45.70 \\
$2-1,133$ & 132.34 & 1.877 & 25.41 & 47.70 \\
$2-2,43$ & 132.94 & 1.556 & 29.82 & 46.40 \\
$2-3,57$ & 134.58 & 1.958 & 24.29 & 47.55 \\
$2-4,61$ & 136.12 & 1.810 & 26.11 & 47.27 \\
$4-1,107$ & 151.08 & 1.899 & 23.21 & 44.07 \\
$4-2,68$ & 152.19 & 1.863 & 19.76 & 36.80 \\
\hline
\end{tabular}

TABLE 4 - Continued

\begin{tabular}{|c|c|c|c|c|}
\hline $\begin{array}{c}\text { Sample } \\
\text { (Interval in } \mathrm{cm} \text { ) }\end{array}$ & $\begin{array}{l}\text { Subbottom } \\
\text { Depth (m) }\end{array}$ & $\begin{array}{l}\text { Density } \\
(\mathrm{g} / \mathrm{cc})\end{array}$ & $\begin{array}{c}\text { Water Content } \\
(\%)\end{array}$ & $\begin{array}{l}\text { Porosity } \\
\text { (\%) }\end{array}$ \\
\hline $5-1,140$ & 160.91 & 1.926 & 21.32 & 41.07 \\
\hline $9-1,108$ & 198.59 & 1.946 & 20.79 & 40.47 \\
\hline $9-2,75$ & 199.76 & 2.155 & 23.46 & 50.55 \\
\hline $9-3,30$ & 200.81 & 1.900 & 20.05 & 38.10 \\
\hline $9-4,46$ & 202.47 & 1.893 & 22.54 & 42.67 \\
\hline $11-1,125$ & 217.76 & 1.844 & 17.58 & 32.40 \\
\hline $11-2,57$ & 218.58 & 2.041 & 24.18 & 49.36 \\
\hline $12-3,57$ & 229.58 & 1.867 & 18.57 & 34.67 \\
\hline $12-4,55$ & 231.06 & 1.953 & 22.76 & 44.44 \\
\hline $12-6,53$ & 234.04 & 2.058 & 21.96 & 45.20 \\
\hline $13-1,55$ & 236.16 & 1.836 & 24.01 & 44.08 \\
\hline $13-2,40$ & 237.51 & 1.773 & 22.79 & 40.40 \\
\hline $13-4,54$ & 240.65 & 1.598 & 22.03 & 35.20 \\
\hline $13-6,57$ & 243.68 & 1.919 & 23.08 & 44.28 \\
\hline $14-4,53$ & 250.29 & 1.932 & 21.93 & 42.37 \\
\hline $15-3,37$ & 257.88 & 1.319 & 22.16 & 29.23 \\
\hline $15-6,53$ & 262.54 & 1.572 & 22.48 & 35.33 \\
\hline $16-2,60$ & 266.11 & 2.079 & 23.33 & 48.50 \\
\hline $16-3,55$ & 267.56 & 1.830 & 24.01 & 43.93 \\
\hline $17-2,57$ & 275.58 & 1.842 & 24.54 & 45.20 \\
\hline $17-4,55$ & 287.56 & 1.976 & 22.49 & 44.44 \\
\hline $18-2,56$ & 285.07 & 1.696 & 22.81 & 38.68 \\
\hline $18-4,57$ & 288.08 & 1.543 & 23.41 & 36.12 \\
\hline $19-2,57$ & 294.58 & 1.855 & 21.38 & 39.67 \\
\hline \multicolumn{5}{|l|}{ Site 374} \\
\hline $2-1,109$ & 158.10 & 1.802 & 29.84 & 53.77 \\
\hline $2-2,76$ & 159.27 & 1.865 & 25.95 & 48.40 \\
\hline $2-3,64$ & 160.65 & 1.860 & 29.70 & 55.23 \\
\hline $3-1,110$ & 209.11 & 1.936 & 24.71 & 47.83 \\
\hline $4-2,86$ & 253.87 & 1.908 & 23.19 & 44.23 \\
\hline $4-3,107$ & 255.58 & 1.914 & 26.92 & 51.52 \\
\hline $4-4,35$ & 256.36 & 1.901 & 27.10 & 51.53 \\
\hline $6-1,67$ & 331.63 & 1.918 & 22.32 & 42.80 \\
\hline
\end{tabular}

Site 376

$\begin{array}{lrrrr}1-1,105 & 1.06 & 1.751 & 34.88 & 61.06 \\ 1-3,54 & 3.55 & 1.566 & 40.11 & 62.80 \\ 2-1,100 & 8.51 & 1.657 & 34.04 & 56.40 \\ 2-4,57 & 12.58 & 1.727 & 33.60 & 58.03 \\ 3-2,122 & 19.73 & 1.648 & 35.53 & 58.57 \\ 3-5,110 & 24.11 & 1.720 & 34.32 & 59.03 \\ 5-3,45 & 39.46 & 1.733 & 28.01 & 48.53 \\ 5-4,82 & 41.33 & 1.754 & 32.71 & 57.37 \\ 7-1,100 & 56.01 & 1.878 & 24.83 & 46.63 \\ 8-1,96 & 65.47 & 1.951 & 25.63 & 50.00 \\ 8-3,141 & 68.92 & 1.962 & 24.28 & 47.63 \\ 9-1,96 & 74.97 & 1.906 & 27.89 & 53.17 \\ 9-3,55 & 77.56 & 2.023 & 27.08 & 54.77 \\ 10-1,87 & 84.38 & 1.873 & 19.93 & 37.33 \\ 10-3,111 & 87.62 & 1.918 & 21.91 & 42.03 \\ 11-2,71 & 95.22 & 1.896 & 23.56 & 44.60 \\ 12-3,60 & 106.11 & 1.912 & 27.32 & 52.23 \\ 13-3,106 & 116.07 & 1.677 & 24.47 & 41.03 \\ 13-3,117 & 116.18 & 2.047 & 19.25 & 39.40 \\ 15-2,81 & 133.32 & 1.959 & 20.35 & 39.85\end{array}$

Site 377

$\begin{array}{lllll}1-1,86 & 191.37 & 1.775 & 33.13 & 58.80 \\ 1-2,80 & 192.81 & 1.898 & 31.06 & 58.93\end{array}$

Hole 378

$\begin{array}{lrrrr}2-4,52 & 98.53 & 1.841 & 28.97 & 53.32 \\ 3-1,123 & 104.24 & 1.730 & 33.92 & 58.70 \\ 3-2,73 & 105.24 & 1.725 & 33.12 & 57.13 \\ 6-2,65 & 143.16 & 1.721 & 30.47 & 52.43 \\ 7-1,55 & 170.06 & 1.865 & 27.24 & 50.80 \\ 8-1,56 & 217.57 & 1.784 & 30.89 & 55.10\end{array}$

Hole 378A

$\begin{array}{lllll}\mathrm{i}-4,52 & 51.03 & 1.678 & 34.81 & 58.40\end{array}$


TABLE 5

\begin{tabular}{lccc}
\hline $\begin{array}{c}\text { Sample } \\
\text { (Interval in cm) }\end{array}$ & $\begin{array}{c}\text { Density } \\
(\mathrm{g} / \mathrm{cc})\end{array}$ & $\begin{array}{c}\text { Water Content } \\
(\%)\end{array}$ & $\begin{array}{c}\text { Porosity } \\
(\%)\end{array}$ \\
\hline & & & \\
Site 371 & & & \\
& & & \\
$1-4,75$ & 1.70 & 36.8 & 62.0 \\
$2-3,75$ & 2.00 & 23.4 & 43.6 \\
$3-5,70$ & 1.98 & 24.9 & 45.0 \\
$3-6,73$ & 2.02 & 25.6 & 47.2 \\
$4-5,75$ & 2.12 & 23.4 & 48.6 \\
5-6, 63 & 2.11 & 28.4 & 50.9 \\
6, CC & - & 20.1 & -
\end{tabular}

Site 372

\begin{tabular}{|c|c|c|c|}
\hline $1-1,143$ & 1.77 & 32.9 & 56.0 \\
\hline $1-4,18$ & 1.99 & 30.2 & 49.8 \\
\hline $2-3,49$ & 1.98 & 26.2 & 49.9 \\
\hline $3-1,66$ & 1.90 & 28.6 & 52.2 \\
\hline $4-1,79$ & 1.93 & 25.9 & 47.9 \\
\hline $11-3,25$ & 1.98 & 26.1 & 48.7 \\
\hline $12-5,30$ & 1.94 & 24.1 & 44.4 \\
\hline $14-4,16$ & 1.90 & 25.6 & 46.3 \\
\hline $15-5,54$ & - & 25.6 & - \\
\hline $16-5,100$ & - & 25.3 & - \\
\hline $17-5,91$ & - & 24.4 & - \\
\hline $18-5,100$ & - & 24.8 & - \\
\hline $20-6,28$ & - & 25.6 & - \\
\hline $21-6,117$ & - & 24.7 & - \\
\hline $22-4,96$ & - & 24.9 & - \\
\hline $23-4,56$ & - & 24.7 & - \\
\hline $24-6,29$ & - & 25.2 & - \\
\hline $26-3,58$ & - & 23.2 & - \\
\hline $27-5,53$ & - & 19.0 & - \\
\hline $28-5,47$ & - & 22.6 & - \\
\hline $29-4,99$ & - & 25.4 & - \\
\hline $30-5,15$ & - & 23.5 & - \\
\hline $31-5,39$ & - & 21.0 & - \\
\hline $31-4,128$ & - & 28.0 & - \\
\hline $32-4,28$ & - & 23.3 & - \\
\hline $32-5,114$ & - & 22.5 & - \\
\hline $32-6,100$ & - & 21.5 & - \\
\hline $33-1,50$ & - & 23.2 & - \\
\hline $33-4,77$ & - & 22.6 & - \\
\hline $34-2,20$ & - & 22.4 & - \\
\hline $34-3,141$ & - & 19.8 & - \\
\hline $35-2,14$ & - & 18.6 & - \\
\hline $35-3,58$ & - & 20.9 & - \\
\hline $36-4,136$ & - & 20.0 & - \\
\hline $37-2,135$ & - & 20.6 & - \\
\hline $38-1,134$ & - & 19.1 & - \\
\hline $38-5,15$ & - & 19.4 & - \\
\hline $39-1,12$ & - & 19.5 & - \\
\hline $39-3,34$ & - & 19.5 & - \\
\hline $40-2,77$ & - & 19.3 & - \\
\hline $40-3,01$ & - & 20.4 & - \\
\hline $41-4,14$ & - & 20.0 & - \\
\hline $41-6,83$ & - & 17.5 & - \\
\hline $42-1,119$ & - & 18.4 & - \\
\hline $43-2,70$ & - & 16.2 & - \\
\hline $44-1,119$ & - & 16.8 & - \\
\hline $45-3,141$ & - & 14.8 & - \\
\hline $46-3,06$ & - & 16.1 & - \\
\hline \multicolumn{4}{|l|}{ te 394} \\
\hline $2-2,74$ & 1.88 & 26.9 & 48.6 \\
\hline $3-1,74$ & 1.96 & 30.8 & 56.5 \\
\hline $4-3,58$ & 2.01 & 25.5 & 46.8 \\
\hline $5-2,87$ & 2.11 & 30.1 & 55.3 \\
\hline $5-3,105$ & 2.01 & 29.6 & 50.7 \\
\hline
\end{tabular}

TABLE 5 - Continued

\begin{tabular}{lccc}
\hline $\begin{array}{c}\text { Sample } \\
\text { Interval in cm) }\end{array}$ & $\begin{array}{c}\text { Density } \\
(\mathrm{g} / \mathrm{cc})\end{array}$ & $\begin{array}{c}\text { Water Content } \\
(\%)\end{array}$ & $\begin{array}{c}\text { Porosity } \\
(\%)\end{array}$ \\
\hline & & & \\
$5-4,11$ & 2.08 & 28.3 & 50.2 \\
$5-5,29$ & - & 31.1 & - \\
$6-4,31$ & 1.96 & 31.3 & 52.4 \\
$6-5,31$ & - & 34.2 & - \\
$6-6,78$ & 1.96 & 32.4 & 54.4 \\
$7-4,51$ & 2.10 & 31.1 & 51.4 \\
$7-5,40$ & 2.01 & 31.7 & 50.9 \\
$7-6,102$ & 2.02 & 25.6 & 42.4 \\
$8-3,27$ & 2.14 & 29.9 & 49.9 \\
$8-4,91$ & 2.01 & 25.7 & 41.6 \\
$9-4,71$ & - & 22.4 & - \\
$10-1,118$ & 2.18 & 22.5 & 29.5 \\
$12-2,71$ & - & 32.3 & - \\
$13-2,72$ & 2.10 & 18.1 & 30.7 \\
$14-2,88$ & 2.07 & 20.7 & 33.6 \\
$15-2,37$ & 2.14 & 20.4 & 33.0 \\
$19-1,66$ & - & 21.5 & -
\end{tabular}

Site 375

$\begin{array}{llrl}4-4,9 & - & 15.2 & - \\ 7-4,63 & - & 17.0 & - \\ 9-3,23 & - & 9.0 & - \\ 10-2,71 & - & 12.2 & -\end{array}$

Site 376

$\begin{array}{lccc}1-2,32 & - & 38.3 & - \\ 1-4,41 & - & 39.5 & - \\ 2-2,79 & - & 40.8 & - \\ 2-3,68 & - & 34.5 & - \\ 3-3,94 & 1.73 & 32.8 & 55.4 \\ 3-4,32 & 1.75 & 34.3 & 58.5 \\ 5-2,101 & - & 31.4 & - \\ 5-5,12 & - & 33.1 & - \\ 6-3,81 & 1.79 & 33.1 & 57.7 \\ 6-4,21 & - & 31.0 & - \\ 8-2,111 & 2.01 & 17.0 & 33.4 \\ 9-2,31 & - & 29.0 & - \\ 9-3,16 & - & 33.6 & - \\ 10-2,120 & - & 20.1 & - \\ 11-3,26 & - & 30.5 & - \\ 12-2,129 & - & 28.6 & - \\ 12-4,42 & - & 23.2 & - \\ 13-2,94 & - & 29.6 & - \\ 13-4,100 & - & 30.9 & - \\ 15-3,14 & 1.92 & 22.1 & 41.3 \\ 16-1,47 & 1.76 & 23.0 & 39.2\end{array}$

Site 378

\begin{tabular}{llll}
$1-1,75$ & - & 34.43 & - \\
$2-5,75$ & - & 31.02 & - \\
$3-3,105$ & - & 32.58 & - \\
$6-3,50$ & - & 31.26 & - \\
$7-3,44$ & - & 31.05 & - \\
$8-2,97$ & - & 36.23 & - \\
\hline
\end{tabular}

TABLE 6

\begin{tabular}{lc}
\hline $\begin{array}{c}\text { Sample } \\
\text { (Interval in cm) }\end{array}$ & $\begin{array}{c}\text { Shear } \\
\text { Strength }\left(\mathrm{kg} / \mathrm{cm}^{2}\right)\end{array}$ \\
\hline Site 371 & \\
& \\
$1-4,110$ & 0.045 \\
$2-3,110$ & 0.579 \\
\hline
\end{tabular}


TABLE 6 - Continued

\begin{tabular}{cc}
\hline $\begin{array}{c}\text { Sample } \\
\text { (nterval in cm) }\end{array}$ & $\begin{array}{c}\text { Shear } \\
\text { Strength }\left(\mathrm{kg} / \mathrm{cm}^{2}\right)\end{array}$ \\
\hline & \\
$3-6,110$ & 0.660 \\
$4-5,110$ & 1.401
\end{tabular}

Site 372

\begin{tabular}{ll}
$1-3,110$ & 0.230 \\
$1-4,129$ & 0.481 \\
$1-4,135$ & 0.488 \\
$2-2,108$ & 0.349 \\
$2-3,16$ & 0.376 \\
$2-3,27$ & 0.598 \\
$2-4,37$ & 0.411 \\
$3-1,61$ & 0.517 \\
$3-1,65$ & 0.407 \\
$3-2,48$ & 0.334 \\
$3-3,127$ & 0.437 \\
$4-1,90$ & 0.712 \\
$4-1,131$ & 0.963 \\
$4-2,79$ & 0.823 \\
$9-2,15$ & 1.149 \\
$9-4,88$ & 1.263 \\
$10-2,102$ & 2.913 \\
$10-2,133$ & 1.066 \\
$11-3,38$ & 0.938 \\
$12-5,30$ & 0.851 \\
$14-4,42$ & 1.307 \\
\hline
\end{tabular}

TABLE 7

\begin{tabular}{ccc}
\hline $\begin{array}{c}\text { Sample } \\
\text { (Interval in cm) }\end{array}$ & $\begin{array}{c}\text { Subbottom } \\
\text { Depth }(\mathrm{m})\end{array}$ & $\begin{array}{c}\text { Thermal } \\
\text { Conductivity } \\
\left(\mathrm{mcal} / \mathrm{cm} \mathrm{sec}^{\circ} \mathrm{C}\right)\end{array}$ \\
\hline
\end{tabular}

Site 371

$\begin{array}{lrr}1-4,130 & 5.80 & 3.205 \\ 1-4,140 & 5.90 & 3.495 \\ 2-3,45 & 202.95 & 3.480 \\ 3-4,114 & 366.74 & 3.301 \\ 3-6,155 & 369.85 & 3.532 \\ 4-3,90 & 412.40 & 3.737 \\ 4-3,110 & 412.60 & 3.120 \\ 4-6,130 & 417.30 & 4.330 \\ 5-6,15 & 473.30 & 3.600 \\ 5-6,30 & 473.45 & 3.671 \\ 5-6,45 & 473.55 & 3.338\end{array}$

Site 372

\begin{tabular}{lll}
$1-3,120$ & 116.20 & 3.102 \\
$2-4,20$ & 135.20 & 3.114 \\
$3-2,55$ & 142.55 & 3.334 \\
$3-3,70$ & 144.20 & 3.177 \\
$4-2,60$ & 152.10 & 3.349 \\
$10-2,99$ & 209.49 & 3.276 \\
$11-3,48$ & 219.98 & 3.099 \\
$12-5,29$ & 233.79 & 3.376 \\
$13-5,76$ & 242.26 & 3.142 \\
$13-6,29$ & 243.29 & 3.619 \\
$14-2,41$ & 246.91 & 3.548 \\
$14-4,50$ & 250.00 & 3.477 \\
$15-5,136$ & 261.86 & 3.332 \\
$16-5,59$ & 270.59 & 3.409 \\
$17-5,60$ & 280.10 & 3.618 \\
$18-5,30$ & 289.30 & 3.552 \\
$19-5,89$ & 299.39 & 3.622 \\
$20-6,45$ & 310.95 & 3.487 \\
\hline
\end{tabular}

TABLE 7 - Continued

\begin{tabular}{ccc}
\hline $\begin{array}{c}\text { Sample } \\
\text { (Interval in cm) }\end{array}$ & $\begin{array}{c}\text { Subbottom } \\
\text { Depth }(\mathrm{m})\end{array}$ & $\begin{array}{c}\text { Thermal } \\
\text { Conductivity } \\
\left(\mathrm{mcal} / \mathrm{cm} \mathrm{sec}^{\circ} \mathrm{C}\right)\end{array}$ \\
\hline
\end{tabular}

\section{Hole 373A}

$\begin{array}{lrr}1-1,127 & 97.77 & 2.255 \\ 1-2,116 & 99.16 & 2.071 \\ 1-3,80 & 100.30 & 2.089\end{array}$

Site 374

$\begin{array}{lll}1-2,107 & 103.70 & 2.922 \\ 2-2,106 & 159.56 & 2.895 \\ 2-3,106 & 161.06 & 2.632 \\ 4-2,26 & 253.26 & 3.385 \\ 4-3,110 & 255.60 & 3.063 \\ 4-4,114 & 257.14 & 2.804 \\ 5-2,109 & 299.59 & 3.177 \\ 5-2,119 & 299.69 & 3.007 \\ 5-3,110 & 301.10 & 3.412 \\ 5-3,121 & 301.21 & 3.284 \\ 5-4,85 & 302.35 & 3.085 \\ 5-4,101 & 302.51 & 3.286 \\ 5-5,103 & 304.03 & 3.226 \\ 5-5,111 & 304.11 & 3.209 \\ 6-4,108 & 336.08 & 3.112 \\ 6-5,58 & 337.08 & 2.601 \\ 6-6,108 & 339.08 & 2.509 \\ 7-5,92 & 346.92 & 3.369 \\ 8-3,54 & 353.04 & 3.339\end{array}$

Site 376

$\begin{array}{lrl}1-2,90 & 2.40 & 2.161 \\ 1-4,83 & 5.33 & 2.335 \\ 2-2,89 & 9.89 & 2.485 \\ 2-3,75 & 11.25 & 2.801 \\ 3-4,62 & 22.12 & 2.798 \\ 4-2,62 & 28.62 & 2.753 \\ 5-2,73 & 38.23 & 2.721 \\ 5-2,82 & 38.32 & 2.826 \\ 5-2,86 & 38.36 & 2.853 \\ 5-2,126 & 38.76 & 2.848 \\ 6-3,82 & 49.32 & 2.996 \\ 6-4,77 & 50.77 & 2.878 \\ 7-2,88 & 57.38 & 2.894 \\ 8-2,91 & 66.91 & 2.767 \\ 9-2,75 & 76.25 & 2.758 \\ 9-3,91 & 77.91 & 3.226 \\ 10-2,79 & 85.79 & 3.127 \\ 12-2,63 & 104.63 & 3.020 \\ 12-4,63 & 107.63 & 3.001 \\ 13-2,78 & 114.28 & 2.996 \\ 13-4,90 & 116.59 & 2.922\end{array}$

Site 377

$\begin{array}{lll}1-2,77 & 192.77 & 2.986\end{array}$

Hole 378

$\begin{array}{lll}2-5,90 & 100.40 & 2.974 \\ 3-3,89 & 106.89 & 2.939 \\ 5-2,95 & 124.45 & 2.648 \\ 6-3,88 & 144.88 & 2.618 \\ 7-3,92 & 173.42 & 2.800 \\ 11-3,85 & 306.35 & 3.211\end{array}$

Hole 378A

\begin{tabular}{lrr}
$1-5,82$ & 52.82 & 2.836 \\
$3-5,101$ & 300.01 & 3.625 \\
\hline
\end{tabular}

